\title{
Uso do eye tracking para obtenção de medidas quantitativas em testes de usabilidade: Um estudo focado na medida da satisfação
}

The use of eye tracking to obtain quantitative measurements in usability tests: a study focused on the measurement of the satisfaction

\author{
Aline Girardi Gobbi ${ }^{1}$ \\ Eugenio Andrés Díaz Merino ${ }^{2}$ \\ Giselle Schmidt Alves Díaz Merino ${ }^{3}$ \\ Leila Amaral Gontijo ${ }^{4}$
}




\section{Resumo}

As três principais métricas adotadas para a avaliação da usabilidade são a eficácia, a eficiência e a satisfação. Porém, a satisfação é usualmente medida por meio de questionários, entrevistas ou protocolos verbais, o que gera resultados subjetivos e/ou dados qualitativos que podem não ser confiáveis. O eye tracking é uma ferramenta que vêm sendo utilizada em testes de usabilidade, trazendo dados quantitativos e, portanto, resultados mais precisos. O objetivo desta pesquisa é verificar se e como estão sendo feitas as medidas de satisfação utilizando o eye tracking. Para cumprir com este objetivo, foi realizada uma Revisão Sistemática nas principais bases de dados internacionais e nacionais, na busca por artigos publicados em periódicos e revisados por pares. Foram encontrados 90 resultados sobre este tema na data da pesquisa, porém, percebe-se que há um interesse crescente na área e algumas indicações de como os dados obtidos por meio do eye tracking podem ser utilizados para medir a satisfação do usuário em testes de usabilidade.

Palavras-chave: Usabilidade, Satisfação, Eye Tracking.

\section{Abstract}

Effectiveness, efficiency and satisfaction are the three key metrics used to evaluate usability. However, satisfaction is usually measured through questionnaires, interviews or verbal protocols, which generates subjective results and / or qualitative data that may not be reliable. Eye tracking is a tool that has been used in usability tests, bringing quantitative data and, therefore, more accurate results. The objective of this research is to verify if eye tracking can be used to measure satisfaction and how can it be done. In order to comply with this objective, a Systematic Review was conducted in the main international and national databases, in the search for articles published in periodicals and peer reviewed. We found 90 results on this topic at the time of the survey, however, it is noticed that there is a growing interest in the area and some indications of how the data obtained through eye tracking can be used to measure user satisfaction in usability tests.

Key-words: Usability, Satisfaction, Eye Tracking.

ISSN: 2316-7963

\footnotetext{
${ }^{1}$ Mestra em Design, Doutoranda em Engenharia - UFSC (aline.gobbi@gmail.com)

2 Prof. Dr. Universidade Federal de Santa Catarina - UFSC

${ }^{3}$ Prof. Dra. Universidade Federal de Santa Catarina - UFSC

${ }^{4}$ Prof. Dra. Universidade Federal de Santa Catarina - UFSC
} 


\section{Introdução}

Em 2001, Han et al. afirmavam que os usuários estavam cada vez mais intolerantes com relação às dificuldades no uso de produtos, dando preferência aos que são fáceis de usar e de aprender. Essa percepção continua atual e até mesmo mais importante diante do mercado que se tornou ainda mais competitivo, visto que com o uso massivo da internet, e consequentemente, o acesso à informação, os usuários trocam informações e percepções sobre produtos continuamente. Neste contexto, produtos difíceis de usar causam frustração e desconforto no usuário que adquiriu o produto, e essa experiência, quando compartilhada, pode trazer grandes prejuízos às empresas que lançaram o produto.

Diante deste cenário, os testes de usabilidade se tornam ainda mais importantes como parte do processo de desenvolvimento de produtos. Acosta et al. (2011) corrobora esta afirmação, quando diz que as empresas já identificam a usabilidade como um fator estratégico para a competitividade, eficiência, diferenciação de produtos e boa prática.

O conceito de usabilidade foi desenvolvido principalmente para a aplicação na interação humano-computador (HCI) (FALCÃO e SOARES, 2013), sendo considerada como um importante atributo para o sucesso de interfaces gráficas (JORDAN, 1998; HAN ET AL., 2001; TULLIS E ALBERT, 2008), e também para produtos físicos.

Os testes de usabilidade são feitos utilizando-se de diversas ferramentas e métricas. Na definição de usabilidade adotada pela norma ISO 9241:11 (1998), as três principais métricas adotadas para a avaliação da usabilidade são a eficácia, geralmente medida pela avaliação do número de tarefas que o usuário conseguiu completar com sucesso, a eficiência, onde usualmente toma-se por base o tempo que o usuário leva para cumprir seus objetivos na tarefa, e a satisfação, medida por meio de questionários, entrevistas ou protocolos verbais (think aloud).

Durante o desenvolvimento de um teste de usabilidade é possível que informações relacionadas com a satisfação do usuário não sejam percebidas. De acordo com Kretschmar et al. (2013), as medições relativas à satisfação são limitadas a um comportamento observável evidente. Através da utilização de entrevistas, questionários ou protocolos think aloud, os pesquisadores devem confiar na memória de um participante e julgamentos subjetivos como um meio de obter insights sobre os processos cognitivos internos e estados emocionais. Um achado comum dentro da neurociência cognitiva é que a percepção subjetiva da pessoa sobre seu próprio comportamento nem sempre corresponde com a sua atividade neural subjacente. Relacionado ao exposto, de forma simplificada, Bergstram e Schall (2014) afirmam que as pessoas nem sempre sabem o que está acontecendo dentro de suas próprias cabeças ${ }^{1}$.

As tecnologias de medição de sinais psicofisiológicos, como o eye tracking (rastreamento ocular) podem auxiliar na identificação de variações emocionais, gerando dados quantitativos que venham a reforçar informações subjetivas relativas à satisfação, coletadas durante os testes.

\footnotetext{
1 "Simply put, people do not always know what is going on inside their own heads." (BERGSTRAM, J. R.; SCHALL, A. J., 2014).
} 
O objetivo desta pesquisa é analisar os resultados obtidos a fim de identificar se e como os dados provenientes de testes utilizando eye tracking (número de piscadas por unidade de tempo, número de sacadas, caminhos percorridos, fixações, entre outros) estão sendo utilizados para a medida da satisfação. Para atingir este objetivo, pretende-se realizar uma revisão sistemática, que consiste no levantamento de publicações em determinadas bases de dados utilizando as palavras-chaves adequadas. Em seguida, esses dados são analisados e os resultados são apresentados.

\section{REFERENCIAL TEÓRICO}

Para um melhor entendimento do tema abordado, foi apresentada a definição dos principais termos relativos ao tema tratados nesta pesquisa, que estão alinhadas com a visão dos pesquisadores.

\subsection{Usabilidade}

A definição de usabilidade adotada nesta pesquisa é a da Norma ISO 9241/11 (1998), que a define como a medida em que um sistema, produto ou serviço pode ser usado por usuários específicos para se atingir objetivos específicos com eficácia, eficiência e satisfação em um determinado contexto de uso.

Segundo van Kuijk (2010), a definição da ISO 9241:11 foi elaborada de forma detalhada, sendo suficientemente genérica para ser aplicada em todas as fases de uso do produto, além de ser a mais aplicada. Apesar disso, o autor ressalta que em cada caso específico, o avaliador deve utilizar as medições apropriadas para as dimensões propostas (eficácia, eficiência e satisfação).

Nielsen (1993) destaca que a usabilidade possui múltiplos componentes, além da eficácia, eficiência e satisfação e cita outras dimensões, como a facilidade de aprendizado, facilidade de memorização e poucos erros na execução da tarefa.

A usabilidade pode ser medida por meio da aplicação de diversos métodos e técnicas. A escolha dos métodos, técnicas e ferramentas, para medição da usabilidade, depende de diversos fatores, como a fase de desenvolvimento do projeto, a quantidade de recursos disponíveis, tempo disponível, experiência do avaliador, o tipo de interação que o produto apresenta, entre outros (MERINO, G. et al., 2012). Esses métodos e técnicas geram dados que podem ser classificados em objetivos e subjetivos.

De acordo com Falcão e Soares (2013), quanto a este aspecto, pode se classificar as medidas de usabilidade em (NIELSEN, 1993; HAN ET AL., 2001; RAITA \& OULASVIRTA, 2011):

- Medidas operacionais de usabilidade de caráter objetivo: correspondem às variáveis quantitativas relativas ao desempenho, ao tempo necessário para executar a tarefa e à quantidade de erros cometidos pelo usuário na execução desta.

- Medidas objetivas de usabilidade: são medidas relativas aos níveis de conhec- 
imento do usuário. Estas medidas levam em consideração o desempenho do usuário experiente, a capacidade do usuário iniciante de aprender e a capacidade do usuário casual de reaprender a utilizar um determinado produto.

- Medidas subjetivas de usabilidade: Essas medidas são baseadas na experiência e percepção do usuário.

Baseada em Han et al. (2001), a figura 1 (FALCÃO e SOARES, 2013) apresenta a abordagem da usabilidade do produto considerando as dimensões objetivas e subjetivas.

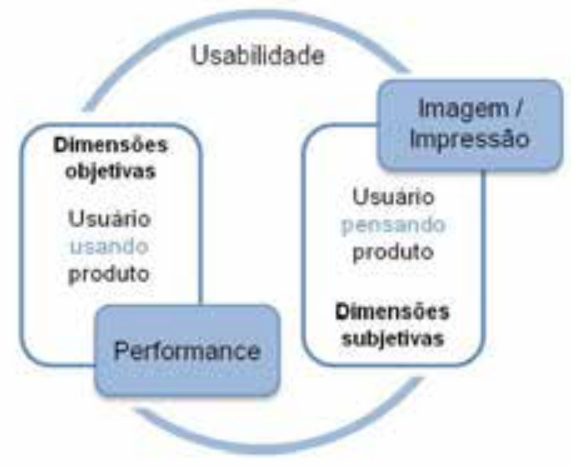

Figura 1: FALCÃO e SOARES (2013), baseado em Han et al. (2001).

As medições relativas à satisfação podem ser encaixadas nas dimensões subjetivas da usabilidade, dado que dependem da percepção do usuário e suas impressões com relação ao produto, conforme será visto no próximo tópico.

\subsection{Satisfação}

Também de acordo com a ISO 9241:11 (1998), a satisfação mede o quanto os usuários estão livres de desconforto, gostam de utilizar o produto ou aceitam a carga mental imposta pela tarefa. A satisfação pode estar relacionada aos objetivos da usabilidade (eficiência e facilidade de aprendizagem).

A satisfação se mostra mais complexa de ser medida, em comparação com a eficácia e a eficiência (de caráter objetivo), por tratar-se de uma medida subjetiva, que depende da percepção dos usuários.

A satisfação pode ser medida por meio de métodos como entrevistas, questionários e até avaliações heurísticas, que abordam algumas questões relacionadas à satisfação, dentro do escopo da usabilidade (GOBBI, 2015). Métodos de medição de satisfação em formato de questionário, como o SUS (System Usability Scale), desenvolvido por John Brooke em 1986, permitem uma aplicação ampla para avaliação de diversos tipos de produtos e sistemas. O método PrEmo (Measure Product Emotion), desenvolvido por Desmet (2002), é também utilizado para medir o sentimento despertado por um produto no usuário. A ferramenta foi atualizada por Caicedo (2009), e consiste em um conjunto de figuras que o participante pode escolher, representando 14 emoções diferentes (ARAÚJO et al., 2015). 
Essas ferramentas podem ser bastante úteis para a medição da satisfação, porém possuem a desvantagem de resultarem em dados subjetivos, o que pode provocar erros de interpretação desses dados.

\subsection{Eye Tracking (Rastreamento Ocular)}

O eye-tracking fornece dados informativos sobre o comportamento da visão do usuário, por meio da captação dos movimentos oculares.

As principais medidas que o eye tracking pode fornecer são:

- Fixação do olhar: A fixação é um momento em que os olhos estão relativamente imóveis (Poole \& Ball, 2005). De acordo com Granka et al. (2008) "a fixação é geralmente definida como um olhar espacialmente estável com duração de aproximadamente 200300 milissegundos, durante o qual a atenção visual é dirigida à uma área específica da exibição visual". No contexto web, as fixações referem-se a informações sobre os elementos da interface que captam a atenção do usuário e por quanto tempo o elemento captou sua atenção. Por meio da análise do local das fixações, também é possível verificar a ordem em que o usuário visualizou cada elemento da interface.

Com relação às fixações do olhar, três medidas podem ser obtidas: 1) Número de fixações, que se refere à quantidade de fixações que são contadas em um determinado período de tempo, ou em uma região específica da tela (interface) ou de um objeto; 2) Tempo de fixação, que consiste no tempo que o participante passou fixando em um mesmo ponto. Segundo alguns autores, quando o tempo de fixação é muito alto, pode significar um aumento da carga cognitiva no usuário (IQBAL et al., 2005; SCHULTHEIS \& JAMESON, 2004) e; 3) Dispersão das fixações, que refere-se à quantidade de fixações afastadas das áreas de interesse.

Com a utilização dos dados relativos às fixações, os softwares de análise de dados do eye tracking são capazes de mapear as fixações em uma tela ou em um cenário, conforme demonstrado na Figura 1. 
Figura 1: Mapa de Fixações (Gazeplot), indicando a ordem das fixações (numérica).

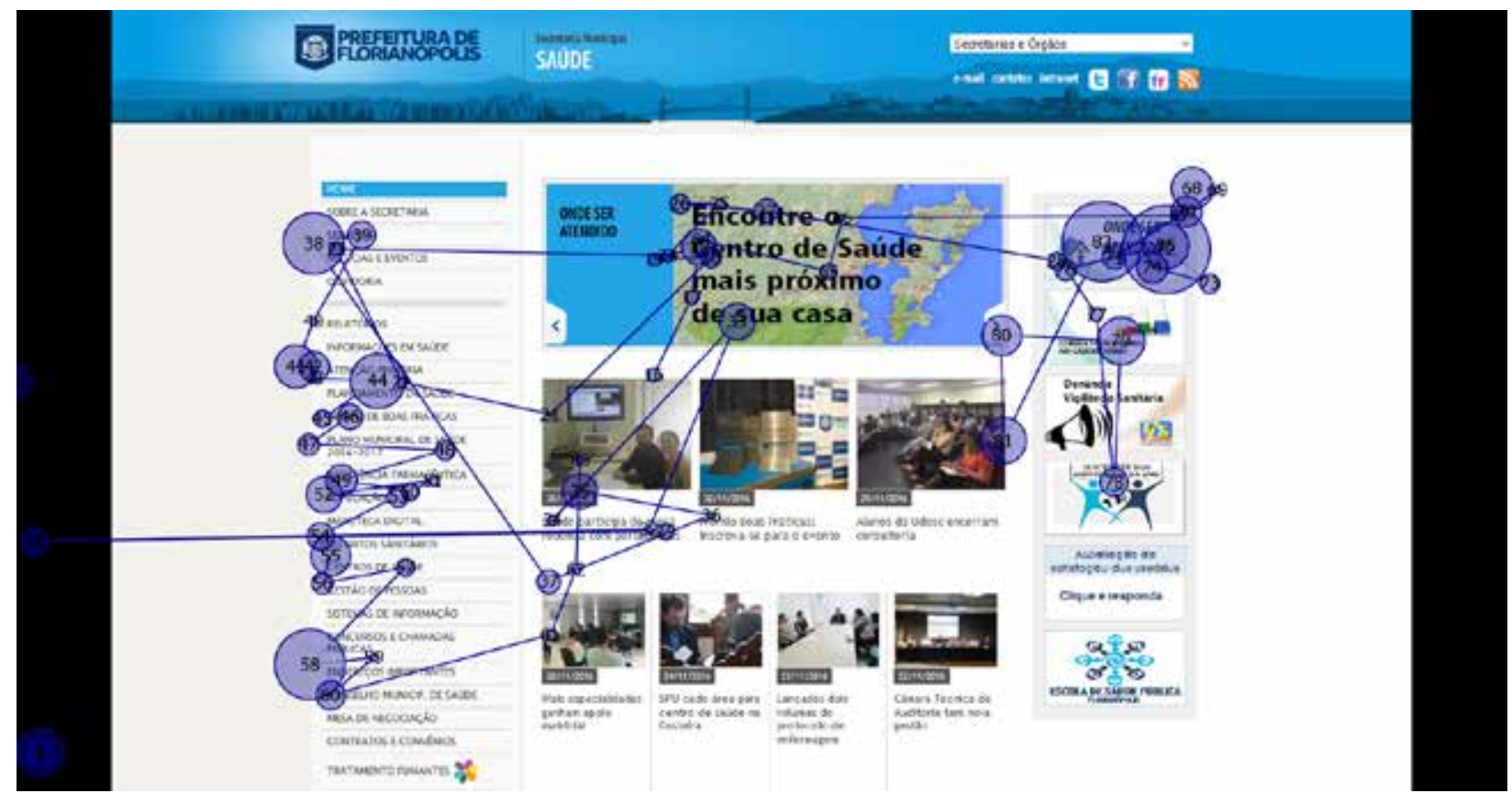

Fonte: Os autores (dados gerados pelos software Beegaze)

- Sacadas: As sacadas ou movimentos sacádicos são explicadas por lida (2016, p. 123):

Durante a leitura ou exame detalhado de diferentes partes de um objeto, o olho não se movimenta continuamente, mas aos "pulos", de uma fixação para outra. Nesse movimento, chamado de sacádico, inicialmente, há uma aceleração na direção desejada, seguida de uma desaceleração e, ao se aproximar do ponto desejado, ocorrem pequenas oscilações para fazer o ajuste fino.

O movimento sacádico, que é baseado nas fixações de um ponto a outro, estabelece o padrão de fixação do olhar e reflete como um usuário interpreta um estímulo visual. Esse padrão fornece uma ideia da hierarquia visual de um cenário. Especificamente, quando se trata de navegação web, o eye tracking pode revelar os princípios da Gestalt no Design no que se refere à ordem em que os elementos de uma página web, por exemplo, são visualizados (BERGSTRAM \& SCHALL, 2014).

- Piscadas: O dipositivo eye tracking retorna o número e a duração das piscadas do participantes durante a execução da tarefa. Tsai et al. (2007) descobriram que a frequência e duração das piscadas corresponde à performance da tarefa, e concluíram que mudanças nas métricas relativas às piscadas eram devidas à carga mental e fadiga do operador, induzidas pela tarefa.

Os resultados da pesquisa de Mcintire et al. (2014) indicaram que as métricas de frequência e duração de piscadas podem ser indicadores da performance de vigilância. Os resultados da frequência de piscadas para um dos olhos e a duração da piscada para ambos os olhos sugere que, à medida que a performance em uma tarefa cai, os participantes piscam com mais frequência, e as piscadas são mantidas por um período de tem- 
po maior, o que pode acarretar em perdas de estímulos visuais importantes (que pode ser devido ao fato de as pálpebras estarem literalmente sendo fechados por mais tempo, fazendo com que faltem os sinais de estímulo, ou devido a simples lapsos; ou ainda a combinação dos dois fatores).

No contexto web, a mudança do número de piscadas durante uma tarefa pode indicar alta carga mental sendo exigida, como por exemplo, encontrar uma informação que esteja escondida em um web site.

\section{PROCEDIMENTOS METODOLÓGICOS}

Conforme o objetivo dessa pesquisa, foi realizada uma revisão sistemática, utilizando o método proposto por Sampaio e Mancini (2007), que descrevem o desenvolvimento da revisão sistemática em cinco etapas:

1. Definir a pergunta;

2. Buscar a evidência;

3. Revisar e selecionar os estudos;

4. Analisar a qualidade metodológica dos estudos;

5. Apresentar os resultados.

Seguindo o método proposto, foi definida a seguinte pergunta de pesquisa: Como estão sendo obtidos os dados para avaliação da satisfação do usuário, por meio da utilização da ferramenta eye tracking?

A busca das evidências se deu em quatro das principais bases de dados internacionais: Web of Science, Scopus, Engineering Village, uma nacional: Scielo. As bases foram selecionadas conforme os seguinte critérios:

- Web of Science: É uma base de dados indexados multidisciplinar que contém o maior número de citações em suas respectivas áreas. Possui mais de 9 mil periódicos indexados (Web of Science, 2016).

- Scopus: É a maior base de dados de resumos e citações da literatura acadêmica. Contém mais de 15 mil periódicos indexados, além de outros documentos (SCOPUS, 2016).

- Enginerring Village: É a base de dados mais completa do mundo dentro dos mais de 190 campos da engenharia, com 20 milhões de literaturas anexadas, provenientes de 77 países (ENGINEERING VILLAGE, 2016)

- Scielo: É a base de maior referência para publicações brasileiras, com 355 periódicos indexados e mais de 300 mil referências (SCIELO, 2016).

Foram analisados todos os artigos provenientes das buscas com a seguinte string: ("eye track*" AND satisfaction), nas revistas internacionais, e na busca na base Scielo foi pesquisado, além da string em inglês, a string em português ("Rastreamento ocular" $\mathrm{E}$ satisfação). 
Foi definido que somente seriam avaliados trabalhos nos idiomas Inglês, português e espanhol, devido à proficiência dos pesquisadores. Também foi definido que seriam excluídos da revisão artigos não encontrados nas plataformas Capes, Google, Google Scholar ou que não fossem fornecidos pelos autores. Somente artigos publicados em revistas e revisados por pares fazem parte da revisão.

As buscas não foram limitadas por data de publicação, de modo a incluir todos os artigos publicados nas bases selecionadas.

Os artigos obtidos foram selecionados considerando sua relação com o tema pesquisado, baseados na análise do título, palavras-chave e resumo dos artigos encontrados. Após a exclusão dos artigos que não têm relação com o tema, foi feita a leitura dos artigos na íntegra, sendo que o critério de seleção dos trabalhos foi o nível de contribuição para responder a pergunta de pesquisa proposta.

\section{RESULTADOS}

Os resultados da Revisão Sistemática foram separados em: Análise Bibliométrica, onde se buscaram dados relativos ao número de publicações por base, número de duplicatas, fator de impacto dos periódicos, entre outros dados considerados relevantes para a análise.

\subsection{Análise Bibliométrica}

A busca foi realizada no dia 25 de outubro de 2016, e foi definido que não seria limitada por data de publicação, de modo a incluir todos os artigos publicados nas bases selecionadas.

Foi pesquisada a string definida ("eye track* AND satisfaction) nas bases internacionais e ("rastreamento ocular" E satisfação) na base nacional obtendo como retorno (números totais, sem aplicação de filtros):

- 154 resultados na base Scopus;

- 92 resultados na base Web of Knowledge;

- 90 resultados na Engineering Village;

- 0 resultados na Scielo.

Todas as bases foram filtradas para mostrar apenas artigos de periódicos revisados por pares, a fim de se obter um resultado mais confiável de artigos para leitura. Desta forma, aplicando o primeiro filtro (apenas periódicos revisados por pares), os resultados totais para cada base foram:

- Scopus: 63 artigos;

- Web of Knowledge: 61 artigos;

- Engineering Village: 16 artigos. 
Após a limitação dos resultados por artigos revisados por pares, foi verificado o número de duplicatas nas quatro bases de dados. Excluindo-se as duplicatas, um total de 90 artigos permaneceu para filtragem posterior, por título, resumo e palavras-chaves. Todos os artigos da base Engineering Village estavam incluídos também na Scopus ou na Web of Knowledge.

Ao final da filtragem por título e resumo, foram selecionados 5 artigos da base Scopus, 4 da Web Of Knowledge e 8 pertencentes às duas bases (duplicatas). Foram excluídos principalmente artigos da área de oftalmologia e medicina, bem como outros não relacionados ao tema pesquisado. Com a leitura na íntegra, foram excluídos 3 artigos que estavam indexados nas duas bases e 1 da Web of Knowledge, por não serem considerados relevantes para esta pesquisa, permanecendo 5 artigos da base Scopus, 5 artigos pertencentes às duas bases e 3 artigos da Web Of Knowledege (Figura 2). Desta forma, a análise com relação ao conteúdo desta revisão, foi realizada com base em 13 artigos no total, distribuídos no Quadro 1.

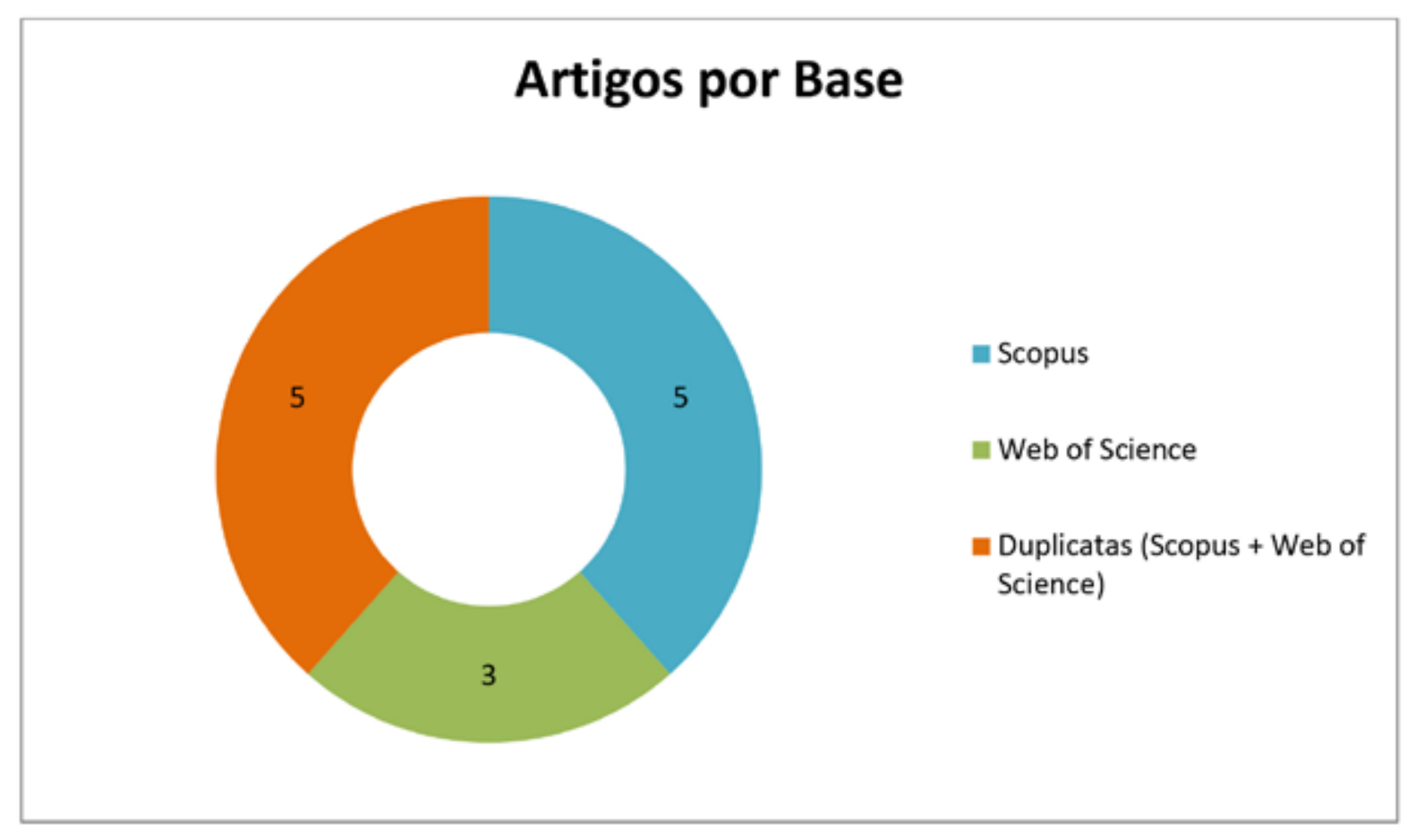

Fonte: os autores. 


\begin{tabular}{|c|c|c|c|c|c|c|}
\hline Base & Periódico & Autores & Titulo & País & Ano & JCR \\
\hline WoK & $\begin{array}{l}\text { IEEE } \\
\text { Transactions } \\
\text { on Systems, } \\
\text { Man, and } \\
\text { Cybernetics }\end{array}$ & $\begin{array}{l}\text { GULLIVER, S. } \\
\text { R.; GHINEA, G. }\end{array}$ & $\begin{array}{l}\text { Stars in Their Eyes: What } \\
\text { Eye-Tracking Reveals About } \\
\text { Multimedia Perceptual } \\
\text { Quality }\end{array}$ & $\begin{array}{l}\text { Reino } \\
\text { Unido }\end{array}$ & 2004 & 1,59 \\
\hline $\begin{array}{l}\text { Scopus } \\
+ \text { WoK }\end{array}$ & $\begin{array}{l}\text { International } \\
\text { Journal of } \\
\text { Human- } \\
\text { Computer } \\
\text { Studies }\end{array}$ & $\begin{array}{l}\text { CYR, D.; HEAD, } \\
\text { M.; LARIOS, H. }\end{array}$ & $\begin{array}{l}\text { Colour appeal in website } \\
\text { design within and across } \\
\text { cultures: A multi-method } \\
\text { evaluation }\end{array}$ & Canadá & 2009 & 1,47 \\
\hline Scopus & $\begin{array}{l}\text { Journal on } \\
\text { Multimodal } \\
\text { User } \\
\text { Interfaces }\end{array}$ & $\begin{array}{l}\text { CHENG, S.; } \\
\text { LIU, Y. }\end{array}$ & $\begin{array}{l}\text { Eye-tracking based adaptive } \\
\text { user interface: implicit } \\
\text { human-computer interaction } \\
\text { for preference indication }\end{array}$ & China & 2012 & 1,01 \\
\hline $\begin{array}{l}\text { Scopus } \\
+ \text { WoK }\end{array}$ & $\begin{array}{l}\text { International } \\
\text { Journal of } \\
\text { Human- } \\
\text { Computer } \\
\text { Interaction }\end{array}$ & $\begin{array}{l}\text { DOHERTY, S.; } \\
\text { O'BRIEN, S. }\end{array}$ & $\begin{array}{l}\text { Assessing the Usability of } \\
\text { Raw Machine Translated } \\
\text { Output: A User-Centered } \\
\text { Study Using Eye Tracking }\end{array}$ & Irlanda & 2013 & 1,26 \\
\hline Scopus & $\begin{array}{l}\text { Journal of } \\
\text { Computer } \\
\text { Science }\end{array}$ & $\begin{array}{l}\text { BAHARUM, A.; } \\
\text { JAAFAR, A. }\end{array}$ & $\begin{array}{l}\text { Evaluation of asean mental } \\
\text { models pattern of web user- } \\
\text { centered interface design } \\
\text { using eye-tracking } \\
\text { technology }\end{array}$ & Malásia & 2014 & $\mathrm{NE}$ \\
\hline Scopus & $\begin{array}{l}\text { The Journal } \\
\text { of Positive } \\
\text { Psychology }\end{array}$ & $\begin{array}{l}\text { SANCHEZ, A.; } \\
\text { VAZQUEZ, C. }\end{array}$ & $\begin{array}{l}\text { Looking at the eyes of } \\
\text { happiness: Positive emotions } \\
\text { mediate the influence of life } \\
\text { satisfaction on attention to } \\
\text { happy faces }\end{array}$ & $\begin{array}{l}\text { Bélgica/ } \\
\text { Espanh } \\
\text { a }\end{array}$ & 2014 & 1,90 \\
\hline $\begin{array}{l}\text { Scopus } \\
+ \text { WoK }\end{array}$ & $\begin{array}{l}\text { Color } \\
\text { Research and } \\
\text { Aplication }\end{array}$ & $\begin{array}{l}\text { CHOI, K.; } \\
\text { SUK, H. }\end{array}$ & $\begin{array}{l}\text { Optimal Employment of Color } \\
\text { Attributes to Achieve } \\
\text { Saliency in Icon Matrix } \\
\text { Desians }\end{array}$ & $\begin{array}{l}\text { Coréia } \\
\text { do Sul }\end{array}$ & 2014 & 0,84 \\
\hline Scopus & $\begin{array}{l}\text { American } \\
\text { Journal of } \\
\text { Distance } \\
\text { Education }\end{array}$ & $\begin{array}{l}\text { SIMUNICH, B.; } \\
\text { ROBINS, D. B.; } \\
\text { KELLY, V. }\end{array}$ & $\begin{array}{l}\text { The Impact of Findability on } \\
\text { Student Motivation, Self- } \\
\text { Efficacy, and Perceptions of } \\
\text { Online Course Quality }\end{array}$ & EUA & 2015 & $\mathrm{NE}$ \\
\hline WoK & $\begin{array}{l}\text { International } \\
\text { Journal of } \\
\text { Industrial } \\
\text { Ergonomics }\end{array}$ & $\begin{array}{l}\text { KHALIGHY, S.; } \\
\text { GREEN, G.; } \\
\text { SCHEEPERS, } \\
\text { C.; WHITTET, } \\
\text { C. }\end{array}$ & $\begin{array}{l}\text { Quantifying the qualities of } \\
\text { aesthetics in product design } \\
\text { using eye-tracking } \\
\text { technology }\end{array}$ & $\begin{array}{l}\text { Reino } \\
\text { Unido }\end{array}$ & 2015 & 1 \\
\hline $\begin{array}{l}\text { Scopus } \\
+ \text { WoK }\end{array}$ & $\begin{array}{l}\text { Computers in } \\
\text { Human } \\
\text { Behavior }\end{array}$ & $\begin{array}{l}\text { CASTILLA, D.; } \\
\text { GARCIA- } \\
\text { PALACIOS, A.; } \\
\text { MIRALLES, I.; } \\
\text { BRETON- } \\
\text { LOPEZ, J.; } \\
\text { PARRA, E. } \\
\text { RODRIGUEZ- } \\
\text { BERGES, S.; } \\
\text { BOTELLA, C. }\end{array}$ & $\begin{array}{l}\text { Effect of Web navigation style } \\
\text { in elderly users }\end{array}$ & $\begin{array}{l}\text { Espanh } \\
\text { a }\end{array}$ & 2015 & 2,8 \\
\hline Scopus & Emotion & RAILA, $H_{\text {.; }}$ & Seeing the World Through & EUA & 2015 & 3,08 \\
\hline
\end{tabular}




\begin{tabular}{|l|l|l|l|l|l|l|}
\hline+ WoK & & $\begin{array}{l}\text { SCHOLL, B. J.; } \\
\text { GRUBER, J. }\end{array}$ & $\begin{array}{l}\text { Rose-Colored Glasses: } \\
\text { People Who Are Happy and } \\
\text { Satisfied With Life } \\
\text { Preferentially Attend to } \\
\text { Positive Stimuli }\end{array}$ & & & \\
\hline Scopus & $\begin{array}{l}\text { On the } \\
\text { Horizon }\end{array}$ & $\begin{array}{l}\text { RENSHAW, T.; } \\
\text { STEVENS, R.; } \\
\text { DENTON, P. D. }\end{array}$ & $\begin{array}{l}\text { Towards understanding } \\
\text { engagement in games: an } \\
\text { eye-tracking study }\end{array}$ & $\begin{array}{l}\text { Reino } \\
\text { Unido }\end{array}$ & 2016 & 0,51 \\
\hline WoK & $\begin{array}{l}\text { Human } \\
\text { Factors and } \\
\text { Ergonomics } \\
\text { in } \\
\text { Manufacturin } \\
\text { g \& Service } \\
\text { Industries }\end{array}$ & $\begin{array}{l}\text { GUO, F.; LIU, } \\
\text { W. L.; CAO, Y.; } \\
\text { LIU, F. T.; LI, M. }\end{array}$ & $\begin{array}{l}\text { Optimization Design of a } \\
\text { Webpage Based on Kansei } \\
\text { Engineering }\end{array}$ & China & 2016 & 0,462 \\
\end{tabular}

Fonte: os autores.

$\mathrm{NE}=\mathrm{Não}$ Encontrado

Os dados do Quadro 1 foram organizados por ano (dos mais antigos para os mais recentes). Neste aspecto, é importante notar que 9 dos 13 artigos foram publicados após 2014, mostrando que esta área de estudos é relativamente nova. O artigo mais antigo data de 2004.

Por ser uma área de estudo relativamente nova, como já mencionado, o fator de impacto (JCR) dos periódicos onde os artigos foram publicados se mostra alto também, variando entre 0,462 e 3,08.

Os artigos são de origem geográfica dispersa, conforme consta no Quadro 1. Vale destacar que 3 dos artigos selecionados são originários do Reino Unido (no primeiro artigo os autores são do Departamento de Sistemas de Informação e Sistemas, Universidade de Brunel e no segundo, os autores são da Universidade de Glasgow e da Universidade de Arte de Glasgow, 2 da China (University of Technology, Centro de Pesquisas da Nokia e Universidade de Northeastern), 2 da Espanha (Universidade Complutense e Jaume I University, Universidade Politécnica de Valência, CIBER of Physiopathology of Obesity and Nutrition) e 2 dos Estados Unidos da América (. Essa análise permite identificar as universidades, laboratórios e autores ao redor do mundo que estão trabalhando nesta área para futuras pesquisas acerca do tema.

\subsection{Análise do Conteúdo dos Artigos Selecionados}

No Quadro 2, é apresentado uma resenha de cada artigo selecionado, juntamente com as palavras-chave, que fornece uma visão geral dos trabalhos encontrados. 


\begin{tabular}{|c|c|c|c|}
\hline Autores & Titulo & Visão geral & Palavras-Chave \\
\hline $\begin{array}{l}\text { BAHARUM, A.; } \\
\text { JAAFAR, A. }\end{array}$ & $\begin{array}{l}\text { Evaluation of asean } \\
\text { mental models pattern } \\
\text { of web user-centered } \\
\text { interface design using } \\
\text { eye-tracking } \\
\text { technology }\end{array}$ & $\begin{array}{l}\text { O estudo utiliza o eye tracking para } \\
\text { verificar a eficiência de web sites de } \\
\text { acordo com o padrão de modelo } \\
\text { mental ASEAN, que afirma que os } \\
\text { usuários tendem a procurar } \\
\text { elementos em locais padrão. }\end{array}$ & $\begin{array}{l}\text { Eye-Tracking, } \\
\text { Mental Models, } \\
\text { Web User- } \\
\text { Centered Interface, } \\
\text { Web Objects, User } \\
\text { Expectation }\end{array}$ \\
\hline $\begin{array}{l}\text { CHENG, S.; LIU, } \\
\text { Y. }\end{array}$ & $\begin{array}{l}\text { Eye-tracking based } \\
\text { adaptive user } \\
\text { interface: implicit } \\
\text { human-computer } \\
\text { interaction for } \\
\text { preference indication }\end{array}$ & $\begin{array}{l}\text { O artigo trata da criação de um } \\
\text { sistema de recomendação de } \\
\text { produtos na web utilizando eye } \\
\text { tracking ao invés de cliques sobre os } \\
\text { produtos de interesse. }\end{array}$ & $\begin{array}{l}\text { Eye-tracking, } \\
\text { Adaptive user } \\
\text { interface, } \\
\text { Preference } \\
\text { inference, Human- } \\
\text { computer } \\
\text { interaction }\end{array}$ \\
\hline $\begin{array}{l}\text { SANCHEZ, A.; } \\
\text { VAZQUEZ, C. }\end{array}$ & $\begin{array}{l}\text { Looking at the eyes of } \\
\text { happiness: Positive } \\
\text { emotions mediate the } \\
\text { influence of life } \\
\text { satisfaction on } \\
\text { attention to happy } \\
\text { faces }\end{array}$ & $\begin{array}{l}\text { O artigo busca relacionar emoções } \\
\text { (bem-estar subjetivo) com aspectos } \\
\text { cognitivos, principalmente a atenção } \\
\text { seletiva. }\end{array}$ & $\begin{array}{l}\text { Attention; Life } \\
\text { Satisfaction; } \\
\text { Positive Mood; } \\
\text { Subjective Well- } \\
\text { Being; Eye } \\
\text { Tracking; } \\
\text { Cognition; } \\
\text { Cognitive Bias; } \\
\text { Selective Attention; } \\
\text { Positive Emotions }\end{array}$ \\
\hline $\begin{array}{l}\text { SIMUNICH, B.; } \\
\text { ROBINS, D. B.; } \\
\text { KELLY, V. }\end{array}$ & $\begin{array}{l}\text { The Impact of } \\
\text { Findability on Student } \\
\text { Motivation, Self- } \\
\text { Efficacy, and } \\
\text { Perceptions of Online } \\
\text { Course Quality }\end{array}$ & $\begin{array}{l}\text { O estudo visa determinar o quanto o } \\
\text { tempo de busca ou a facilidade de } \\
\text { "findability" impacta o aprendizado de } \\
\text { estudantes em ambientes online, } \\
\text { utilizando o eye tracking. }\end{array}$ & - \\
\hline $\begin{array}{l}\text { RENSHAW, T.; } \\
\text { STEVENS, R.; } \\
\text { DENTON, P. D. }\end{array}$ & $\begin{array}{l}\text { Towards } \\
\text { understanding } \\
\text { engagement in games: } \\
\text { an eye-tracking study }\end{array}$ & $\begin{array}{l}\text { Os pesquisadores buscam entender } \\
\text { como os jogadores interagem com } \\
\text { um jogo. Exploram uma variedade de } \\
\text { técnicas (incluindo o eye tracking) } \\
\text { para explorar os insights dos } \\
\text { jogadores no que se refere a } \\
\text { interação, satisfação do usuário, } \\
\text { engajamento e imersão. }\end{array}$ & $\begin{array}{l}\text { Indoor games, E- } \\
\text { learning, Eyes, } \\
\text { Tracking }\end{array}$ \\
\hline $\begin{array}{l}\text { GUO, F.; LIU, W. } \\
\text { L.; CAO, Y.; LIU, } \\
\text { F. T.; LI, M. L. }\end{array}$ & $\begin{array}{l}\text { Optimization Design of } \\
\text { a Webpage Based on } \\
\text { Kansei Engineering }\end{array}$ & $\begin{array}{l}\text { Utiliza Kansei Engineering, que } \\
\text { incorpora o apelo emocional em } \\
\text { tecnologias ergonomicamente } \\
\text { centradas no usuário, para melhoria } \\
\text { de páginas web. }\end{array}$ & $\begin{array}{l}\text { Kansei engineering; } \\
\text { Webpage } \\
\text { optimization design; } \\
\text { Eye tracking; } \\
\text { Neural network; } \\
\text { Genetic algorithm } \\
\end{array}$ \\
\hline $\begin{array}{l}\text { KHALIGHY, S.; } \\
\text { GREEN, G.; } \\
\text { SCHEEPERS, C.; } \\
\text { WHITTET, C. }\end{array}$ & $\begin{array}{l}\text { Quantifying the } \\
\text { qualities of aesthetics } \\
\text { in product design } \\
\text { using eye-tracking } \\
\text { technology }\end{array}$ & $\begin{array}{l}\text { O estudo tem a pretensão de } \\
\text { quantificar qualidades estéticas } \\
\text { objetivamente utilizando um } \\
\text { framework baseado no uso do eye } \\
\text { tracking. }\end{array}$ & $\begin{array}{l}\text { Aesthetics; Product } \\
\text { design; Eye- } \\
\text { tracking; Design } \\
\text { methodology; } \\
\text { Design tool }\end{array}$ \\
\hline $\begin{array}{l}\text { GULLIVER, S. R.; } \\
\text { GHINEA, G. }\end{array}$ & $\begin{array}{l}\text { Stars in Their Eyes: } \\
\text { What Eye-Tracking } \\
\text { Reveals About } \\
\text { Multimedia Perceptual } \\
\text { Quality }\end{array}$ & $\begin{array}{l}\text { Considerações sobre a qualidade de } \\
\text { multimídias utilizando eye tracking e } \\
\text { medidas subjetivas. }\end{array}$ & $\begin{array}{l}\text { Eye tracking, frame } \\
\text { rate, multimedia } \\
\text { video, Quality-of- } \\
\text { Perception (QoP). }\end{array}$ \\
\hline
\end{tabular}




\begin{tabular}{|c|c|c|c|}
\hline Autores & Titulo & Visão geral & Palavras-Chave \\
\hline $\begin{array}{l}\text { DOHERTY, S.; } \\
\text { O'BRIEN, S. }\end{array}$ & $\begin{array}{l}\text { Assessing the } \\
\text { Usability of Raw } \\
\text { Machine Translated } \\
\text { Output: A User- } \\
\text { Centered Study Using } \\
\text { Eye Tracking }\end{array}$ & $\begin{array}{l}\text { Realizado teste de usabilidade de } \\
\text { tradutores virtuais utilizando eye } \\
\text { tracking. Foi indicada uma relação } \\
\text { entre longos tempos de tarefa, alto } \\
\text { número de fixações e duração média } \\
\text { como indicativo de carga cognitiva. }\end{array}$ & - \\
\hline $\begin{array}{l}\text { CASTILLA, D.; } \\
\text { GARCIA- } \\
\text { PALACIOS, A.; } \\
\text { MIRALLES, I.; } \\
\text { BRETON- } \\
\text { LOPEZ, J.; } \\
\text { PARRA, E. } \\
\text { RODRIGUEZ- } \\
\text { BERGES, S.; } \\
\text { BOTELLA, C. }\end{array}$ & $\begin{array}{l}\text { Effect of Web } \\
\text { navigation style in } \\
\text { elderly users }\end{array}$ & $\begin{array}{l}\text { Estudo comparativo entre navegação } \\
\text { linear e hipertextual com usuários } \\
\text { idosos utilizando eye tracking. }\end{array}$ & $\begin{array}{l}\text { Usability, Web } \\
\text { navigation, Elderly, } \\
\text { Web design }\end{array}$ \\
\hline $\begin{array}{l}\mathrm{CHOI}, \mathrm{K} \text {; , SUK, } \\
\mathrm{H} .\end{array}$ & $\begin{array}{l}\text { Optimal Employment } \\
\text { of Color Attributes to } \\
\text { Achieve Saliency in } \\
\text { Icon Matrix Designs }\end{array}$ & $\begin{array}{l}\text { O Objetivo da pesquisa é verificar } \\
\text { quais cores chamam mais atenção } \\
\text { do usuário, utilizando o eye tracker. }\end{array}$ & $\begin{array}{l}\text { Color saliency; } \\
\text { Interface displays; } \\
\text { Icon matrix; Hue; } \\
\text { Tone; Color } \\
\text { combination }\end{array}$ \\
\hline $\begin{array}{l}\text { RAILA, H.; } \\
\text { SCHOLL, B. J.; } \\
\text { GRUBER, J. }\end{array}$ & $\begin{array}{l}\text { Seeing the World } \\
\text { Through Rose-Colored } \\
\text { Glasses: People Who } \\
\text { Are Happy and } \\
\text { Satisfied With Life } \\
\text { Preferentially Attend to } \\
\text { Positive Stimuli }\end{array}$ & $\begin{array}{l}\text { A pesquisa foca na possibilidade de } \\
\text { conexão entre felicidade e } \\
\text { mecanismos cognitivos, } \\
\text { especialmente a atenção visual. }\end{array}$ & $\begin{array}{l}\text { Attention, } \\
\text { Happiness, Eye } \\
\text { tracking }\end{array}$ \\
\hline $\begin{array}{l}\text { CYR, D.; HEAD, } \\
\text { M.; LARIOS, H. }\end{array}$ & $\begin{array}{l}\text { Colour appeal in } \\
\text { website design within } \\
\text { and across cultures: A } \\
\text { multi-method } \\
\text { evaluation }\end{array}$ & $\begin{array}{l}\text { Estudo sobre o apelo das cores em } \\
\text { páginas web, levando em } \\
\text { consideração diferentes culturas. Foi } \\
\text { demonstrado que o tempo de fixação } \\
\text { em determinados esquemas de } \\
\text { cores é maior quando os usuários } \\
\text { gostam do esquema. }\end{array}$ & $\begin{array}{l}\text { Colour appeal; } \\
\text { Culture; Web site } \\
\text { design; Multi- } \\
\text { methodology; Eye- } \\
\text { tracking }\end{array}$ \\
\hline
\end{tabular}

Fonte: os autores.

De acordo com o Quadro 2, é possível afirmar que os estudos com eye tracking são bem variados no que se refere ao tema pesquisado, o objeto de estudo e às conclusões dos dados analisados. As medidas mais utilizadas são as fixações, seguidas pelos caminhos do olhar (gazepaths) e sacadas. Nenhum dos artigos pesquisados foca na questão das piscadas (número de piscadas ou intervalo entre piscadas), embora existam autores que relacionem essas métricas com a satisfação (Hou et al., 2015).

Outro aspecto analisado nos artigos é a definição da satisfação e suas formas de medição. Alguns autores (DOHERTY \& O'BRIEN, 2013; CASTILLA, D. et al., 2015) estão de acordo com Nielsen e Levy (1994), autores consagrados na área de usabilidade, que indicam que os usuários preferem sistemas em que sua performance é melhor, o que significa que quando um produto é eficaz e eficiente, automaticamente ele trará satisfação ao usuário.

Já de acordo com Norman (2007), outro autor consagrado da área de usabilidade, apenas a eficácia e a eficiência não são indicativos de satisfação do usuário. 0 usuário pode ter conseguido completar as tarefas com facilidade e em um período de tempo razoável, mas isso não significa que o mesmo sentiu-se satisfeito ao utilizar o produto. $O$ autor trata mais profundamente do campo das emoções humanas relacionadas à interação com produtos e serviços. Nesse sentido, alguns autores estu- 
dados nessa revisão sistemática vão de acordo com o pensamento de Norman, como CYR; HEAD \& LARIOS (2009), SANCHEZ \& VAZQUEZ (2014), que relacionam emoções (bem-estar subjetivo) com aspectos cognitivos (atenção seletiva).

Apesar de o objetivo inicial deste artigo ter sido a obtenção de medidas de movimento ocular utilizando eye tracking para medição da satisfação, as pesquisas em que a satisfação não foi medida diretamente por meio dos dados obtidos com o eye tracking nao foram excluídos desta pesquisa, primeiramente por não ter sido encontrado, nesta revisão, um artigo que medisse diretamente a satisfação por meio do eye tracking, sendo esta medição feita apenas por inferências. Outro motivo, é que muitas pesquisas trazem dados que podem ser interessantes para pesquisas futuras ou algumas sugestões iniciais do que poderia ser medido para avaliar a satisfação utilizando o eye tracker.

Com relação ao uso do eye tracking para medição direta da satisfação, foi verificado que muitos artigos utilizam o eye tracking em testes de usabilidade apenas para medir eficiência e satisfação, ou outros dados relativos à cognição, interesse visual e atenção mas continuam usando questionários para medir a satisfação, especificamente (CASTILLA et al., 2015; BAHARUM \& JAAFAR, 2014; CHENG \& LIU, 2011; SIMUNICH, ROBINS \& KELLY, 2015; RENSHAW, STEVENS \& DENTON, 2009; GUO et al., 2016). Alguns dos artigos buscam fazer algumas inferências a partir dos resultados do eye tracking referente a algumas medições mais subjetivas como engajamento (RENSHAW, STEVENS \& DENTON, 2009), interesse (CHENG \& LIU, 2011) e preferência (CHOI \& SUK, 2014).

Em alguns dos artigos pesquisados, existe uma preocupação em obter dados quantitativos para a medida da satisfação. Conforme citado por RENSHAW, STEVENS \& DENTON (2009), os pesquisadores envolvidos na medida da satisfação têm usado entrevistas e questionários, potencialmente tendo resultados problemáticos e subjetivos devido à degeneração da memória ou a reconstrução racional da memória feita pelo usuário. Essa observação, feita pelos autores em 2009, ainda parece ser válida, conforme demonstrado nos artigos analisados. Ainda de acordo com os mesmos autores, a medição dos movimentos oculares pode se mostrar útil na medição da satisfação, afirmando que os olhos têm sido fortemente associados com as emoções.

Alguns autores cujos trabalhos não foram encontrados nessa revisão sistemática, como Renshaw (2004) e Hou et al. (2015), já mostraram que pode ser possível medir a satisfação por meio dos movimentos oculares. RENSHAW (2004) contou o número de regressões do olhar (quando o usuário fixa o olhar em pontos já fixados anteriormente). Os resultados indicaram que existe uma relação entre a quantidade de regressões e a satisfação, sendo que quanto menor o número de regressões, mais alto seria o nível de satisfação do usuário. Hou et al. (2015) encontrou relações entre o intervalo entre as piscadas dos usuários na execução de uma tarefa com a satisfação percebida pelo usuário. Essas pesquisas demonstram que o trabalho de medir satisfação com eye tracking parece estar ainda no início, assim como demonstrado por meio desta revisão sistemática, fato que abre um campo de possibilidades de estudo na área. 


\section{CONCLUSÃO}

As principais métricas, indicadas pela ISO 9241:11 (1998), para a avaliação da usabilidade são a eficácia, a eficiência e a satisfação. A satisfação é usualmente medida por meio de questionários, entrevistas ou protocolos verbais, que geram resultados subjetivos que podem não ser confiáveis. O eye tracking é uma ferramenta que vêm sendo utilizada, mais recentemente, conforme se demonstrou nos resultados desta pesquisa, em testes de usabilidade, trazendo dados quantitativos e resultados mais precisos.

A Revisão Sistemática feita nesta pesquisa com a string de busca ("eye track*" AND satisfaction), ou ("rastreamento ocular" E satisfação), em português, tinha como objetivo verificar como estavam sendo feitas medições de satisfação, em testes de usabilidade, utilizando eye tracking. Especificamente em testes de usabilidade não foi encontrado nenhum estudo que medisse a satisfação de forma direta por meio dos dados obtidos com o eye tracking neste estudo, por este motivo, foram analisados artigos que tratassem da medição da satisfação com o eye tracking mesmo que de forma indireta (por meio das medidas relacionadas à eficácia, eficiência, atenção, cognição, preferências e interesses).

Os resultados desta pesquisa mostraram que o estudo dos movimentos oculares para a medição da satisfação em testes de usabilidade é ainda escasso, sendo, portanto, uma área de estudo pouco explorada até o momento.

Como visto na análise dos artigos, o eye tracking pode ser uma ferrramenta poderosa para a obtenção de dados qualitativos na área de usabilidade e seu potencial parece ter sido pouco explorado. Isso pode ser explicado por aspectos como custos e disponibilidade do equipamento ou problemas no uso de tecnologias no design. Surge então uma oportunidade de pesquisa aplicada, com o desenvolvimento de testes de usabilidade por meio dos movimentos oculares, que dão indícios de ser bons indicativos do comportamento do usuário.

O Núcleo de Gestão de Design (NGD), localizado na Universidade Federal de Santa Catarina (UFSC) possui o equipamento eye tracking da SMI (SensoMotoric Instruments), que vem sendo utilizado atualmente em pesquisas de usabilidade, na área de Design, com equipamentos e-reader (SCHMIDT et al., 2016), para testes de usabilidade de interfaces (GOBBI et al., 2017), avaliação de embalagem (LOCKS, 2016), e ainda trabalhos em andamento na área de engenharia civil, especificamente na área de conforto visual e ofuscamento.

Essas pesquisas que vêm sendo realizadas no NGD, assim como as futuras pesquisas a serem feitas, tanto no próprio NGD como em outros grupos de pesquisa ao redor do mundo podem dar início a estudos mais aprofundados sobre os movimentos oculares, possibilitando resultados quantitativos e, portanto, mais precisos nas avaliações de usabilidade. 


\section{AGRADECIMENTOS}

Agradecemos ao CNPq pelo financiamento dos bolsistas envolvidos nesta pesquisa e ao NGD (Núcleo de Gestão de Design) e LDU (Laboratório de Design e Usabilidade) pela disponibilização do laboratório.

\section{REFERÊNCIAS}

ACOSTA, G.; MORALES, K.; LAGOS, D.; ORTIZ, M. Addressing Human Factors and Ergonomics in Design Process, Product Life Cycle, and Innovation: Trends in Consumer Product Design. In: Karwowski, W; Soares, M and Stanton, N. (eds) Human Factors and Ergonomics in Consumer Product Design: Methods and Techniques. CRC Press, 133-154, 2011.

ARAÚJO, F. S. Avaliação da experiência do usuário: uma proposta de Sistematização para o processo de desenvolvimento de produtos. Tese de Doutorado. UFSC, Florianopolis, 2014.

BAHARUM, A.; JAAFAR, A. Evaluation of ASEAN mental models pattern of web user-centered interface design using eye-tracking technology. Journal of Computer Science, v. 10, n. 12, p. 2494, 2014.

BERGSTRAM, J. R.; SCHALL, A. J. Eye Tracking in User Experience Design. Elsevier, 2014.

CASTILLA, D.; Garcia-Palacios, A.; Miralles, I.; Breton-Lopez, J.; Parra, E.; Rodriguez-Berges, S.; Botella, C. Effect of Web navigation style in elderly users. Computers in Human Behavior, v. 55, p. 909-920, 2016.

CHENG, S.; LIU, Y. Eye-tracking based adaptive user interface: implicit human-computer interaction for preference indication. Journal on Multimodal User Interfaces, v. 5, n. 1-2, p. 77-84, 2012.

CHOI, K.; SUK, H. Optimal employment of color attributes to achieve saliency in icon matrix designs. Color Research \& Application, v. 40, n. 5, p. 429-436, 2015.

CYR, D.; HEAD, M.; LARIOS, H. Colour appeal in website design within and across cultures: A multi-method evaluation. International journal of human-computer studies, v. 68, n. 1, p. 1-21, 2010.

DOHERTY, S.; O'BRIEN, S.. Assessing the usability of raw machine translated output: A user-centered study using eye tracking. International Journal of Human-Computer Interaction, v. 30, n. 1, p. 40-51, 2014. 
IIDA, I.; BUARQUE, L. Ergonomia: Projeto e Produção. São Paulo: Blucher, 2016.

ISO 9241-11: Ergonomic requirements for office work with visual display terminals (VDTs). Part 11 - Guidelines for specifying and measuring usability. Gènève: International Organisation for Standardisation, 1998.

ENGINEERING VILLAGE. Disponível em www.engineeringvillage.com. Acesso em set. 2016.

FALCÃO, C.; SOARES, M. Usabilidade de Produtos de Consumo: uma análise dos conceitos, métodos e aplicações. Estudos em Design, v. 21, n. 2, 2013.

GRANKA, L.; FEUSNER, M.; LORIGO, L. Eye monitoring in online search. In R. I. Hammoud, editor, Passive Eye Monitoring, Signals and Communication Technology, pages 347-372. Springer, 2008.

GOBBI, A. G. Desenvolvimento e implementação do MD3E (método de desdobramento em 3 etapas) em ambiente virtual: projeto e avaliação de interface com foco nos fatores humanos. Dissertação de Mestrado. UFSC, 2015.

GOBBI, A. G.; CATECATI, T.; MERINO, E. A. D.; MERINO, G. S. A. D.; FERREIRA, M. G. G. Uso do eye tracking para medição da satisfação para testes de usabilidade em interfaces web. Anais do 16 ERGODESIGN, USIHC e CINAHPA, Edgard Blucher Proceedings: São Paulo, 2017.

GULLIVER, S. R.; GHINEA, G. Stars in their eyes: what eye-tracking reveals about multimedia perceptual quality. IEEE Transactions on Systems, Man, and Cybernetics-Part A: Systems and Humans, v. 34, n. 4, p. 472-482, 2004.

GUO, F.; LIU, W. L.; CAO, Y.; LIU, F. T.; LI, M. L. Optimization design of a webpage based on Kansei engineering. Human Factors and Ergonomics in Manufacturing \& Service Industries, v. 26, n. 1, p. 110-126, 2016.

HAN, S.H.; YUN, M.H.; KWAHK, J.; HONG, S.W. Usability of consumer electronic products. International Journal of Industrial Ergonomics 28:143 - 151, 2001.

HOU, W.; YANG, J.; LYU, F; LI, T. User Satisfaction Evaluation Model Based on Blink Interval. In: International Conference on Human Centered Computing. Springer International Publishing, 2014. p. 477-487.

KHALIGHY, S.; GREEN, G.; SCHEEPERS, C.; WHITTET, C. Quantifying the qualities of aesthetics in product design using eye-tracking technology. International Journal of Industrial Ergonomics, v. 49, p. 31-43, 2015. 
LOCKS, R. T. A contribuição da gestão de design no projeto de embalagem. Dissertação de Mestrado. UFSC, 2016.

MCINTIRE, L.; K, MCKINLEY; GOODYEAR, C.; MCINTIRE, J. Detection of vigilance performance using eye blinks. Applied ergonomics, v. 45, n. 2, p. 354-362, 2014.

MERINO, G. S. A. D.; TEIXEIRA, C. S., SCHOENARDIE, R. P., MERINO, E. A. D.; GONTIJO, L. A. Usability in Product Design - The importance and need for systematic assessment models in product development - Usa - Design Model (U-D)@. Work, v. 41, n. Supplement 1, p. 1045-1052, 2012.

NIELSEN, J. Usability Engineering. Boston: Academic Press, 1993.

NIELSEN, J.; LEVY, J.(1994).Measuring usability: preference vs. performance. Communications of the ACM,37(4),66-75, 1994.

NORMAN, D. A. Design emocional: por que adoramos (ou detestamos) os objetos do dia-a-dia. Rocco, 2008.

POOLE, A., BALL, L.J. Eye tracking in human-computer interaction and usability research: current status and future prospects. In: Ghaoui, C. (Ed.), Encyclopedia of Human Computer Interaction. Idea Group, Hershey, PA, pp. 211-219, 2005.

RAILA, H.; SCHOLL, B. J.; GRUBER, J. Seeing the world through rose-colored glasses: People who are happy and satisfied with life preferentially attend to positive stimuli. Emotion, v. 15, n. 4, p. 449, 2015.

RAITA, E.; OULASVIRTA, A. Too good to be bad: Favorable product expectations boost subjective usability ratings. Interacting with Computers 23: 363-371, 2011.

RENSHAW, T.; STEVENS, R.; DENTON, P. D. Towards understanding engagement in games: An eye-tracking study. On the Horizon, v. 17, n. 4, p. 408-420, 2009.

RENSHAW, J.A. Designing for visual influence: an eye tracking study. Tese de Doutorado. Leeds Metropolitan University, Leeds, 2004.

SANCHEZ, Al.; VAZQUEZ, C. Looking at the eyes of happiness: Positive emotions mediate the influence of life satisfaction on attention to happy faces. The Journal of Positive Psychology, v. 9, n. 5, p. 435-448, 2014.

SCHMIDT, M. C.; MARTINS, D. G.; RODRIGUES, R. S., GOBBI A. G.; MERINO, G. S. A. D.; FERREIRA, M. G. G. Desempenho do e-reader LEV: uma análise utilizando a ferramenta eye tracking. Human Factors in Design, v. 5, n. 10, 2016.

SCIELO. Disponível em: http://www.scielo.org. Acesso em set. 2016. 
SCOPUS. Disponível em http://www.scopus.com. Acesso em set. 2016.

SIMUNICH, B.; ROBINS, D. B.; KELLY, V.. The Impact of Findability on Student Motivation, Self-Efficacy, and Perceptions of Online Course Quality. American Journal of Distance Education, v. 29, n. 3, p. 174-185, 2015.

TSAI, Y., VIIRRE, E., STRYCHACZ, C., CHASE, B., JUNG, T. Task performance and eye activity: predicting behavior relating to cognitive workload. Aviat. Space Environ. Med. 78 (5.2), B176eB185, 2007.

TULLIS, T.; ALBERT, B. Measuring the user experience: collecting, analyzing and presenting usability metrics. USA: Elsevier Inc., 2008.

VAN KUIJK, J.; CHRISTIAANS, H; KANIS, H.; VAN EIJK, D. Usability in the Development of Consumer Electronics: Issues and Actors. Proceedings of 16th World Congress on Ergonomics, Maastricht, The Netherlands, 2006.

WEB OF SCIENCE. Disponível em: http://www.webofknowledge.com. Acesso em set. 2016. 